\title{
Why Embrace Refractive Cataract Surgery?
}

\author{
David F Chang, MD
}

Clinical Professor, University of California, San Francisco

\begin{abstract}
The author comments on the challenges in becoming a 'refractive' cataract surgeon and why restoring the value-added proposition to patients ultimately promotes, rewards, and sustains excellence in cataract surgery.
\end{abstract}

\section{Keywords}

Multifocal intraocular lens (IOL), Toric IOL, refractive IOL, Medicare, cataract surgery

Disclosure: David F Chang, MD, is Clinical Professor at the University of California, San Francisco, and in private practice in Los Altos, California. He donates consulting fees and royalties from Alcon, $\mathrm{AMO}$, and EyeMaginations to Project Vision and the Himalayan Cataract Project.

Received: July 13, 2011 Accepted: August 1, 2011 Citation: US Ophthalmic Review, 2011;4(2):74-5 DOI: 10.17925/USOR.2011.04.02.74

Correspondence: David F Chang, MD, 762 Altos Oaks Drive, Los Altos, California 94024. E: dceye@earthlink.net

The concept of refractive cataract surgery became officially recognized and validated in the US following the 2005 Centers for Medicare and Medicaid (CMS) ruling allowing ophthalmologists to bill Medicare patients separately for presbyopia-correcting intraocular lenses (IOLS). This formalized a very critical distinction in the minds of patients, surgeons, and third-party payors. Health insurance covers medically necessary cataract surgery with a monofocal IOL. Any additional expenses incurred for refractive benefits are not medically necessary and therefore become the option and responsibility of the patient. Astigmatic keratotomy, toric IOLs, and presbyopia-correcting IOLs all fall under the elective refractive channel, which may also include using the femtosecond laser for astigmatic keratotomy.

More than six years later, implantation rates of so called 'premium' IOLS in the US still remain below $15 \%$ (14 \% in Q1 2011, of which approximately one-half were toric IOLS). ${ }^{1}$ Is patient demand this limited, or are there other major reasons for this small of a market share? Although cost is undoubtedly a barrier for many patients and others are poor candidates, there are still many surgeons who are not comfortable, interested, or confident enough to encourage appropriate patients to elect some or all of these options.

Certainly, cataract surgeons continue to covet better refractive IOL technologies. However, I believe that many cataract surgeons have been discouraged by the prospect of transforming how they practice and manage cataract patients. If we think about it, we cataract surgeons have been spoiled by the many advances in cataract surgery, whose results routinely exceed patient expectations. Patients arrive at our offices worried about their declining vision and are fearful of surgery. The favorable risk-benefit ratio is easy to explain, the operation is covered by health insurance, and the procedure and recovery are quick. After surgery, we are accustomed to consistently exceeding most patients' expectations. The speed and comfort of the operation and recovery period surprise most patients-no bandage, no restrictions, no redness, pain, or bruising. Whom among us ever tires of hearing our patients marvel at how clear, bright, and colorful the world now appears, how quickly their vision has improved, and how much better they see without spectacles? What other common operation so regularly results in near-instant gratification?

Much of this potentially changes with refractive IOLS, because the patient must now pay a significant elective out-of-pocket cost. This creates more of a consumer mentality and a need to hear and ask questions about all of the options. There are confusing optical concepts to understand-accommodation, focal distance, monovision, astigmatism, visual aberrations, optical quality and contrast sensitivity. Some patients become hopelessly confused. Patients usually need more time to reach a decision and want to research the options over the Internet or by talking with others. For ophthalmologists not accustomed to a cosmetic or refractive patient population, there are the awkward logistics of having to discuss fees with patients. Most importantly, as we all know, it is easy for patients to develop specific expectations that are not eventually fulfilled. Inflated expectations arise from boastful friends, from company educational materials, from the Internet, from the power of suggestion, and from a desire to simplify the proposition: 'so if I pay more for the better lens, I won't need any glasses, right?'

With refractive cataract surgery, the lengthier discussions, the heightened expectations, and the inevitable increase in dissatisfied patients pose a stark contrast to what we are accustomed to with conventional cataract surgery. It is therefore understandable why many ophthalmologists would rather not deal with the increased chair time, pressure and demands of implanting premium refractive IOLS. The most important reason to embrace refractive cataract surgery is that many patients want and 
should be given these options. So many motivated patients are grateful for and thrilled with their reduced spectacle dependence. However, I believe there is another important reason that ophthalmologists should embrace the opportunity to provide refractive cataract surgery. Premium refractive IOLS are the patient value-added proposition that restores proper value to our skills as cataract surgeons and clinicians.

Andy Corley has pointed out that when control of the doctor-patient relationship was transferred to third-party insurers, the stage was set for the most common procedures such as cataract surgery to become commoditized. Cataract surgery is one of the most successful operations in all of medicine, with more than a $98 \%$ success rate. Furthermore, we have done such a good job of training cataract surgeons that affordable quality surgery is available to virtually all who require it in developed nations. According to cost utility studies, cataract surgery has a present value of $\$ 95,000$, which is higher than that for other common and costlier treatments such as for breast cancer $(\$ 20,000)$ or myocardial infarction $(\$ 70,000)$. ${ }^{2}$

In business, value is determined by what someone would be willing to trade for that service or product-in this case, an operation to halt and permanently reverse a progressive condition that is the leading cause of global blindness. However, governments are the primary purchasers of cataract surgery, which by virtual of its procedural volume is the number one line item expense in the Medicare budget. CMS is not the beneficiary of the surgery and must use quantifiable metrics to determine its relative value. As we all know, the resource-based relative value scale (RB-RVS) system uses overhead costs and procedural time to compare different medical procedures. Ever since this valuation methodology was introduced in 1992, and against the background of continuous budgetary pressures, there has been a $42 \%$ decrease in Medicare's reimbursement for cataract surgery, without adjusting for inflation.

Our patients have been removed from the value proposition of cataract surgery and the resulting insurance payments are far below what the true value for permanently reversing a progressive blinding condition should be. Furthermore, our rising national debt and the aging 'Baby
Boomer' generation assure that maintaining even the current procedural reimbursement is not sustainable.

With the commoditization of cataract surgery, the decline in reimbursement has incentivized both physicians and facilities to lower overhead and increase procedural volume. This is a disincentive to spending greater time with patients and to developing and incorporating newer and more expensive technology for the office and operating room.

We all owe much to Andy Corley's bold vision when, as Chief Executive Officer of Eyeonics, he championed the premium IOL channel as a way to restore the value proposition directly to patients. By differentiating between medical and refractive benefits, and removing insurance payors from this aspect of the doctor-patient relationship, the person deriving the benefit is able to determine the value of the service. Unlike fees capped by government health policy, this arrangement financially rewards excellence. Besides the IOL technology, this premium also encompasses the additional testing, evaluation, education, and counseling that must be part of any recommendation about refractive IOLS and services. Based on the additional procedural time needed to perform an astigmatic keratotomy or implant a multifocal IOL, the RVS value for these services would be trivial. Fortunately, patients do not judge value based solely on the incremental surgical time. Besides the technology involved, what patients really value is the surgeon's knowledge, experience, preparation, communication skills, compassion, integrity, and cognitive clinical judgment.

Refractive cataract surgery restores value to clinical and surgical excellence, and compels us to improve in all aspects of the evaluation and treatment of cataract patients. We need to spend more time with, and more effectively educate and counsel, our patients. We must strive for excellence in our office services, in our diagnostic and surgical technology, and with our surgical and interpersonal skills. Compared to most other medical specialties, we are indeed fortunate to be able to differentiate and provide elective services that many of our cataract patients want and value. As refractive IOL technology improves, the demand for and success in delivering this value proposition will only increase.

1. Source: Market Scope, 2011.

2. Cutler DM, McClellan M, Is technological change in medicine worth it?, Health Aff (Millwood), 2001;20:11-29. 